\title{
Neue Rubrik: Wissenschaftliche Kurzmitteilung
}

In dieser Ausgabe können wir Ihnen erstmals eine wissenschaftliche Kurzmitteilungen anbieten. Denn es hat zwei Jahre gedauert, bis ein entsprechendes Manuskript zur Veröffentlichung eingereicht wurde. Doch es ist ja eines der Ziele von HeilberufeSCIENCE, vor allem dem wissenschaftlichen Nachwuchs eine Möglichkeit zur Präsentation der Methodik oder Ergebnisse kleinerer Projekte oder Pilotstudien zu bieten. Insbesondere sollten sich Studierende aus den Studiengängen Pflegemanagement, Pflegepädagogik, Pflegewissenschaften, Gerontologie, Altenpflege und Gesundheitswissenschaften/Public Health angesprochen fühlen, interessante Aspekte aus ihren Abschlussarbeiten auf maximal drei Druckseiten zu präsentieren. Vielleicht wirkt ja dieser erste Beitrag von Grünbeck et al. zur Zusammenarbeit von Pflegekräften und PhysiotherapeutInnen bei der Sturzprophylaxe als Anregung für weitere Einreichungen.

Des Weiteren geht in diese Ausgabe die Arbeit von Sedlak et al. aus Managementsicht auf die Einführung von Stationsservicekräften innerhalb der stationären Krankenpflege ein und betrachtet förderliche und hemmende Aspekte bei dieser organisatorischen Veränderungsmaßnahme. Einen anderen Blick auf den Personaleinsatz wirft der Beitrag von Wundratsch et al., in dem die Ergebnisse einer Untersuchung zu körperlichen Belastungen und Beanspruchungen von pädagogischen Unterrichtshilfen an sächsischen Förderschulen präsentiert werden. Mit dem Thema "Pflegeinformatik in den Ausbildungs- curricula" setzt sich der Artikel von Steffan et al. auseinander und stellte die Ergebnisse einer Analyse aller bundesweit verfügbaren Ausbildungscurricula dar. Die Studie von Wittschier et al. setzt sich mit dem bislang kaum betrachteten Thema der Vermögensübertragung im Alter aus Sicht von Bankberatern auseinander. Sie zeigt auf, dass die konzeptionelle Ausrichtung der Beratung zur Vermögensübertragung unzureichend ist und dass diesbezüglich noch Forschungsbedarf besteht.

Jetzt möchte ich Sie einladen, die vier Artikel und den wissenschaftlichen Kurzbeitrag dieser Ausgabe von HeilberufeSCIENCE kritisch zu lesen und in den wissenschaftlichen Dialog einzutreten. Im November erwarten Sie übrigens zwei Ausgaben von HeilberufeSCIENCE: die reguläre vierte Ausgabe dieses Jahrgangs und eine Sonderausgabe mit den Abstracts der Kongressbeiträge und Poster auf dem 8. Gesundheitspflege-Kongress vom 19. bis 20. November 2010 in Hamburg. Klicken Sie rein, es würde mich freuen.

$\mathrm{Ihr}$

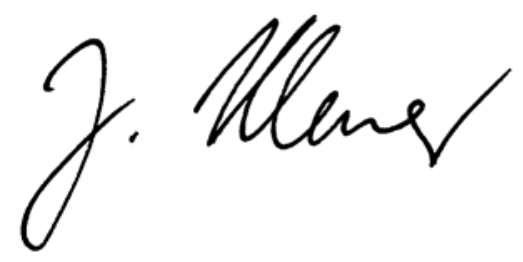

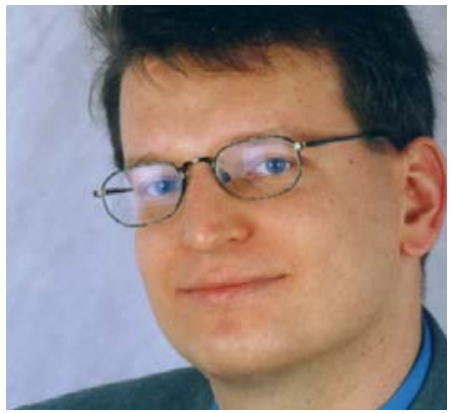

Prof. Dr. med. habil. Jörg Klewer, Zwickau

Schriftleitung

heilberufescience@springer.com

HeilberufeSCIENCE 2010; 1 (3): 72

DOI 10.1007/s16024-010-0314-7 